\title{
TYPOLOGIE LINGUISTIQUE ET DESCRIPTION DES LANGUES EN DANGER
}

\author{
Denis Creissels
}

\section{Résumé}

La description des langues en danger est cruciale pour la typologie linguistique, comme source possible d'information sur des types de structures non attestés dans les langues préalablement décrites. Inversement, une bonne information sur l'état des connaissances en typologie est particulièrement utile aux linguistes qui décrivent des langues jusque là peu décrites, ce qui est le cas de la plupart des langues en danger. Toutefois, les typologues ne doivent pas perdre de vue qu'il y a un problème à mettre sur le même plan, dans la recherche de généralisations typologiques, des langues toujours bien vivantes et largement décrites, et d'autres dont l'unique description est impossible à vérifier ou à compléter. En outre, on ne peut pas travailler sur des langues moribondes comme sur des langues qui sont encore le moyen de communication usuel d'une communauté, et on peut avoir des doutes sur la représentativité des données recueillies dans ces conditions, surtout dans des domaines comme la syntaxe.

\section{Mots-clés}

typologie linguistique, description des langues, linguistique de terrain

\section{Abstract}

The description of endangered languages is crucial for linguistic typology, as a possible source of information about types of structures not attested in the languages described so far. Conversely, good information about the state of knowledge on linguistic typology is particularly useful for linguists dealing with under-described languages, which is generally the case for endangered languages. However, typologists must be conscious that, in the search for typological generalizations, putting on a par well-alive and well-documented languages, and languages whose unique description cannot be checked or completed anymore, may lead to distorted conclusions. Moreover, it is not possible to work on moribund languages in the same way as on languages still used by a community as its usual means of communication, and one may have doubts on the representativity of data collected in such conditions, in particular in domains such as syntax.

\section{Keywords}

linguistic typology, language description, field linguistics 


\section{INTRODUCTION}

Contrairement à ce que peut suggérer l'étymologie de typologie, la typologie linguistique telle qu'on la conçoit actuellement ne consiste pas à essayer de classer les langues en un nombre limité de «types », car il est maintenant bien établi que la diversité des structures linguistiques ne se laisse pas réduire à un tel classement. L'objectif de la typologie linguistique doit plutôt être défini comme la recherche de régularités dans la diversité des structures linguistiques. Il s'agit tout d'abord de dégager des généralisations sur le fait que des modes d'organisation qui seraient a priori envisageables ne sont pas attestés dans les langues du monde (par exemple, dans beaucoup de langues le verbe se place de façon fixe après le premier constituant nominal de la phrase, mais dans aucune langue connue le verbe n'occupe de façon fixe l'avant-dernière position dans la phrase). Mais il s'agit aussi d'observer que certains traits dans l'organisation des langues sont corrélés de façon plus ou moins forte, alors qu'il n'y a aucune nécessité logique à cela (par exemple, les langues qui placent le verbe de façon fixe en fin de phrase marquent généralement la fonction des constituants nominaux au moyen de postpositions plutôt que de prépositions).

L'objectif de cet article est de discuter la relation entre les recherches en typologie linguistique et la description des langues en danger, telle que cette relation s'est établie depuis quelques décennies, et telle qu'on peut prévoir qu'elle va continuer à se développer dans un avenir proche.

\section{L'IMPORTANCE DU TÉMOIGNAGE DES LANGUES PEU ÉTUDIÉES POUR UNE ÉTUDE} SYSTÉMATIQUE DE LA DIVERSITÉ DES STRUCTURES LINGUISTIQUES

Il est évident que la description aussi précise que possible d'une variété aussi large que possible de langues est cruciale pour le développement de la typologie conçue comme la recherche de généralisations à propos de la diversité des structures linguistiques, car la validité des généralisations proposées par la typologie linguistique ne peut pas être évaluée autrement qu'en confrontant ces généralisations à ce que l'on peut observer dans les langues attestées.

Il est évident aussi que plus un trait est rare à travers les langues, et plus la probabilité qu'il soit attesté parmi les langues bien connues des linguistes est faible. Autrement dit, pour avoir une idée aussi exacte que possible de la diversité des structures linguistiques, il importe de ne pas s'arrêter aux langues qui bénéficient d'une tradition grammaticale bien établie, motivée initialement par leur importance sociologique, et de prendre en considération le témoignage d'un maximum de langues sans tenir compte de leur statut social. Il importe notamment de ne pas 
exclure celles dont l'importance pratique est voisine de zéro dans la mesure où elles sont appelées à disparaître dans un avenir relativement proche.

En poursuivant ce raisonnement, on peut argumenter que la description des langues en danger est particulièrement cruciale. En effet, non seulement ces langues, au même titre que toutes les langues non encore décrites, sont susceptibles de permettre de reconnaître des types de structures non attestés dans les langues décrites jusqu'ici, mais en outre leur témoignage risque d'être irrémédiablement perdu si elles ne sont pas décrites avant leur disparition.

On peut d'ailleurs citer divers exemples dans l'histoire de la linguistique, le plus connu étant probablement celui de la découverte de l'ergativité syntaxique en Dyirbal : cette langue australienne, dont l'unique description a été publiée il y a un peu plus de quarante ans, n'a plus qu'un nombre infime de locuteurs (qui sont d'ailleurs plutôt à proprement parler des semi-locuteurs), mais la description qu'en a publié Dixon il y a un peu plus de 40 ans a mis en lumière le fait, largement ignoré jusque là, que certaines langues peuvent avoir des mécanismes syntaxiques dans lesquels c'est le patient des verbes transitifs prototypiques, et non pas l'agent, dont le comportement s'aligne avec celui de l'unique argument des verbes monovalents. Dans un autre domaine, on peut mentionner le système phonologique de l'oubykh, langue caucasique du nord-est qui a été sauvée de l'oubli grâce au travail réalisé par Dumézil avec le dernier locuteur de cette langue. En effet, l'oubykh représente un cas extrême de prolifération de distinctions consonantiques ayant une valeur phonologique, contrastant avec une réduction extrême des distinctions vocaliques.

Plus généralement, il suffit de parcourir n'importe quel ouvrage de typologie pour constater que parmi les phénomènes mentionnés, une bonne proportion est attestée principalement ou même exclusivement dans des langues limitées à des communautés numériquement peu importantes, et de ce fait menacées dans leur existence à plus ou moins brève échéance. Sans prétendre à l'exhaustivité, je mentionnerai ici les systèmes hiérarchiques d'indexation des arguments, les constructions transitives dans lesquelles agent et patient ont une même forme différente de celle de l'argument unique des verbes monovalents, les systèmes de cas spatiaux dans lesquels la forme de locatif s'oppose à une forme unique valant à la fois pour allatif et ablatif, certains types de classificateurs (notamment les classificateurs possessifs), les verbes interrogatifs, etc. etc.

Il y a toutefois d'autres aspects de la question qui doivent eux aussi être pris en considération pour avoir une idée plus précise de la relation entre typologie linguistique et description des langues en danger. 


\section{L'APPORT DE LA TYPOLOGIE LINGUISTIQUE À LA DESCRIPTION}

\section{DES LANGUES EN DANGER}

Dans une réflexion sur la relation entre typologie linguistique et description des langues en danger, il convient de distinguer soigneusement la question de l'apport de la typologie linguistique à la description des langues en danger de celle de l'apport de la description des langues en danger à la typologie linguistique.

En ce qui concerne l'apport de la typologie linguistique à la description des langues en danger, les choses sont simples, car les langues en danger sont très majoritairement des langues jusqu'ici très peu ou pas du tout décrites, et on peut dire à propos de la description des langues en danger la même chose qu'à propos de toutes les langues peu documentées dont on attaque la description, quel que soit leur statut sociologique. En effet, indépendamment du statut social des langues, une bonne information sur l'état des connaissances en typologie est de manière générale utile aux linguistes qui décrivent des langues. Et pour des langues qui ne bénéficient pas d'une tradition grammaticale solidement établie, cette information typologique est particulièrement cruciale, car elle seule permet au descripteur d'avoir une idée des types d'organisation qu'il risque d'être amené à reconnaître dans les données qu'il recueille.

Une bonne information sur la typologie linguistique permet aux linguistes décrivant des langues jusque là sous-décrites de définir une stratégie cohérente et efficace, d'orienter la suite de l'analyse une fois qu'un certain nombre de choses ont été établies, et de mieux évaluer la plausibilité des hypothèses qu'ils peuvent être tentés de formuler. De ce point de vue d'ailleurs, encore plus qu'une information typologique à l'échelle des langues du monde, ce qui est particulièrement utile au linguiste qui décrit une langue sous-décrite, c'est une information typologique aussi approfondie que possible sur les langues qui sont parlées dans la même aire que la langue à décrire ou qui lui sont génétiquement apparentées.

\section{L'APPORT DE LA DESCRIPTION DES LANGUES EN DANGER}

\section{À LA TYPOLOGIE LINGUISTIQUE}

La question de l'apport de la description des langues en danger à la typologie linguistique, et plus particulièrement à la typologie syntaxique, est nettement plus complexe que celle abordée au point 3. Comme ce problème est rarement abordé dans les publications, c'est sur lui que je souhaiterais insister ici.

\subsection{La vulgate}

Pour aborder cette question, on peut prendre comme point de départ le chapitre " Linguistic typology and language documentation » du Oxford Handbook of 
Linguistic Typology (Epps 2010). Ce chapitre est en effet très représentatif du discours « politiquement correct» qui domine sans la moindre nuance tant parmi les typologues que parmi les spécialistes de la documentation des langues en danger. J'entends par là que ce chapitre se borne à développer une évidence, à savoir que l'accumulation de données sur le plus de langues possibles est un facteur essentiel pour les progrès de la typologie linguistique, alors que la typologie linguistique est traditionnellement biaisée en faveur de langues parlées par des communautés relativement importantes et bénéficiant d'une reconnaissance officielle. C'est indiscutable, mais en se limitant à cette argumentation, on évacue en fait des questions gênantes que je souhaite soulever dans ce qui suit.

\subsection{Quelques bémols}

\subsubsection{Deux types de langues en danger}

Dans la discussion qui va suivre, il me semble crucial de distinguer entre des langues en danger qui conservent une certaine vitalité, même si leur avenir est gravement menacé à relativement court terme du fait de changements sociologiques en cours dans la communauté qui les utilisent, et d'autres qui sont déjà à un stade avancé de dépérissement. Bien entendu, il s'agit d'une distinction graduelle et non pas catégorique, ce qui veut dire que bien des langues sont difficiles à classer précisément selon cette distinction. Mais pour ce qui nous occupe ici, il est suffisant d'attirer l'attention sur ce qui différencie les langues qui se situent typiquement dans l'une de ces deux catégories, dans la perspective d'un travail de documentation et de description, et sur les conséquences qu'il serait bon d'en tirer en ce qui concerne l'exploitation des descriptions dans la perspective typologique.

La première catégorie (langues menacées qui conservent néanmoins une certaine vitalité) est celle dans laquelle on peut ranger des langues qui, quelles que soient les menaces qui pèsent sur elles et qui justifient de les classer comme langues en danger, restent encore le moyen de communication quotidien et privilégié pour une partie au moins des locuteurs. À condition de travailler avec des consultants appartenant à cette catégorie, la description de telles langues ne pose pas de problème spécifique par rapport à celle de langues qui ne sont pas considérées comme des langues en danger, et l'utilisation des résultats de la description dans une perspective typologique ne soulève pas de question particulière.

Il n'en va pas de même pour les langues de la deuxième catégorie, c'est-à-dire les langues qui ne restent véritablement un moyen de communication usuel que pour très peu de locuteurs, parfois même aucun. C'est sur les problèmes spécifiques à cette catégorie de langues que je me concentrerai dans ce qui suit. 


\subsubsection{Les difficultés de l'enquête sur des langues à un stade avancé de dépérissement}

Étant donnée une langue à un stade avancé de dépérissement, l'objectif que se fixent généralement les descripteurs (même si cela n'est pas toujours formulé explicitement) est de dégager ce qu'a été le système de la langue en question avant que le processus de dépérissement ne s'engage. Dans cette perspective, il est impératif de privilégier le travail avec des consultants qui ont acquis cette langue à une époque où elle fonctionnait encore comme instrument de communication quotidien et privilégié d'une communauté de locuteurs.

Le choix des consultants pour une description effectuée dans de telles circonstances est loin d'être une question évidente, et même un linguiste expérimenté peut avoir du mal à faire la différence entre des consultants fiables (au sens où ils restituent de manière cohérente la langue qu'ils ont pratiquée dans leur enfance mais qu'ils ne pratiquent plus que de manière occasionnelle), et d'autres qui bricolent avec leurs souvenirs, et dont les productions ne reflètent que de manière très approximative (et parfois à la limite totalement fantaisiste) le système tel qu'il était lorsque la langue avait encore le fonctionnement normal d'une langue. Et pour ajouter à la difficulté, les plus disposés à collaborer avec un linguiste ne sont pas forcément les plus compétents, et on connaît des cas de linguistes qui se sont laissé abuser par des locuteurs auto-proclamés qui se sont avérés ultérieurement n'être que des semi-locuteurs.

Pour éviter les distorsions qui peuvent découler du recours à des consultants qui n'ont pas forcément conservé une pratique active de la langue à décrire, on conseille aux linguistes confrontés à ce type de situation de privilégier le travail sur des textes spontanés, et d'être particulièrement prudents par rapport aux données obtenues en élicitation. Il est incontestable qu'en enregistrant auprès des consultants des histoires traditionnelles, des souvenirs d'enfance et des descriptions de pratiques traditionnelles, on favorise chez eux une restitution aussi fidèle que possible de la langue qu'ils ont pratiquée plus activement dans leur jeunesse. Mais d'une part on n'est jamais totalement à l'abri de distorsions, et ensuite il serait utopique de prétendre se passer totalement de l'élicitation - ou alors, il faudrait se résoudre à laisser sans réponse de nombreuses questions qu'on doit normalement se poser dans la description d'une langue, si le hasard des textes ne fournit pas les données pertinentes.

Un point crucial est que, dans le processus de dépérissement d'une langue, il y $\mathrm{a}$ un stade où les enfants acquièrent encore une certaine compétence dans la langue, qu'ils sont encore capables d'utiliser (au moins jusqu'à un certain point) dans les interactions avec les locuteurs plus âgés, mais où cette acquisition se fait dans des 
conditions très différentes de celle d'une langue pleinement fonctionnelle, avec des conséquences qui ont une incidence directe sur le travail de description. En effet, la manifestation la plus évidente de l'acquisition imparfaite d'une langue qui a déjà largement perdu sa fonctionnalité est la prolifération plus ou moins anarchique de variations (à la fois d'un idiolecte à l'autre et à l'intérieur d'un même idiolecte) plus ou moins dépourvues de cohérence. Tout se passe comme si, à un certain stade de perte de fonctionnalité de la langue au profit d'une autre langue qui tend à se substituer à elle, les locuteurs âgés renonçaient à veiller au respect d'une certaine norme en corrigeant les productions des enfants, comme cela se passe normalement dans une communauté linguistique. Il n'y a rien de surprenant à cela, car la notion de norme perd de son sens pour les derniers locuteurs qui restent capables d'interagir plus ou moins bien avec les locuteurs plus âgés mais n'utilisent plus que marginalement la langue avec leurs pairs.

\subsubsection{Une expérience personnelle}

Dans mon activité de linguiste de terrain, je me suis trouvé confronté à une telle situation lors de mon travail sur l'akhvakh, langue caucasique du nord-est parlée par quelques milliers de locuteurs au Daghestan (Russie) et en Azerbaïdjan. Mes premières enquêtes sur l'akhvakh se sont déroulées dans le village azerbaïdjanais d'Axaxdərə. Ma première impression en arrivant dans ce village n'a pas été celle d'une langue particulièrement menacée : on entendait les parents s'adresser aux enfants en akhvakh, et les enfants répondre aux parents dans la même langue. Mais plus je séjournais à Axaxdərə, et plus je relevais des indices d'une situation beaucoup plus précaire. Je ne mentionnerai ici qu'une anecdote parmi bien d'autres : une jeune femme du village qui avait passé quelques années en ville était venue se réinstaller au village chez ses parents, avec sa fillette âgée de deux ans; du simple fait que cette fillette était née en ville, la famille ainsi que les voisins s'adressaient à elle exclusivement en azerbaïdjanais, et en grandissant la fillette imposait l'usage de l'azerbaïdjanais à tous les enfants (y compris beaucoup plus âgés qu'elle) dont elle partageait les jeux. Plus généralement, plus je progressais dans mon enquête, et plus je me rendais compte que les seuls locuteurs auxquels j'avais facilement accès (les jeunes entre 15 et 25 ans) donnaient seulement l'illusion d'une bonne maitrise de la langue lorsqu'on les observait interagir avec les adultes. L'analyse des textes que j'enregistrais avec eux révélait une prolifération de variations parfois étonnantes, tant entre idiolectes qu'à l'intérieur d'un même idiolecte. Pour ne citer qu'un exemple, alors que l'akhvakh est une langue avec un ordre SOV relativement flexible, l'un de mes consultants produisait des textes avec une prédominance de phrases rangées selon l'ordre SVO. Cette particularité ne pouvait certainement pas s'expliquer par l'influence de l'azerbaïdjanais (langue avec un 
ordre SOV nettement plus rigide que celui de l'akhvakh), et rien dans l'histoire personnelle de ce consultant n'indiquait une exposition possible à une langue sur laquelle il aurait calqué l'ordre SVO que manifestement il affectionnait.

Pour terminer cette anecdote, il me paraît important de préciser que si j'ai fini par y voir à peu près clair dans cette variation (et notamment trier ce qui relevait de fantaisies strictement idiolectales), c'est grâce au travail que j'ai effectué ultérieurement dans les villages akhvakh du Daghestan (où la langue est infiniment mieux conservée, et où la conscience d'une norme est très forte chez les locuteurs) et surtout grâce à l'aide d'Indira Abdulaeva, linguiste daghestanaise elle-même locutrice de l'akhvakh. Ayant passé une semaine à Axaxdərə alors que je m'y trouvais moimême, elle a été immédiatement frappée par le fait qu'une partie des locuteurs âgés (auxquels je n'avais pas réussi à avoir accès moi-même en dépit de nombreuses tentatives) parlaient exactement comme dans les villages du Daghestan, alors que les autres manifestaient ce qu'elle percevait en tant que locutrice native comme des distorsions dépourvues de cohérence.

Pour conclure à propos de l'akhvakh, dans la mesure où on a toujours accès à l'akhvakh « traditionnel » des villages du Daghestan, cela serait certainement très intéressant de faire une étude variationniste des idiolectes d'Axaxdərə. Je l'aurais peut-être fait si les circonstances avaient fait que je continue de m'intéresser aux langues du Caucase. Mais imaginons que l'akhvakh ait cessé d'être parlé ailleurs qu'à Axaxdərə, et que dans trente ans les derniers locuteurs disponibles soient les jeunes dont j'observais les productions entre 2005 et 2008. Il est clair que si on recueille dans trente ans ce qui restera de leurs idiolectes avec comme objectif de reconstituer un système linguistique, le résultat de cette reconstitution n'aura pas grand-chose à voir avec l'akhvakh que leurs grands-parents parlaient encore dans les années 2000 .

4.2.4. La pertinence typologique des descriptions de langues à un stade avancé de dépérissement

Dans cette section, je souhaite attirer l'attention sur les risques que comporte le fait d'injecter sans discrimination dans les bases de données typologiques, notamment dans le domaine de la typologie syntaxique, les données en provenance de langues dont la description n'a pu se faire qu'alors qu'elles se trouvaient déjà à un stade avancé de dépérissement.

Un premier point est qu'il s'agit forcément de données qui seront rapidement invérifiables du fait de la disparition progressive des derniers locuteurs, ce qui veut dire que d'éventuelles distorsions dans les données (distorsions dont les causes peuvent être multiples) seront fixées pour l'éternité. On objectera que cette réserve vaut crucialement pour les descriptions traditionnelles, et beaucoup moins pour les 
descriptions couplées avec un travail systématique de « documentation» au sens donné actuellement à ce terme, mais s'efforcer d'enregistrer tout ce qui peut s'avérer pertinent à l'avenir pour vérifier et éventuellement compléter une description est une entreprise colossale, et dont le résultat n'est tout de même pas vraiment assuré.

Un deuxième point qui me paraît plus fondamental est que, dans une communauté dont la langue est fortement en perte de vitesse, la notion de norme tend à s'estomper pour laisser le champ libre à une prolifération de variations sans commune mesure avec ce qu'on peut observer dans les langues qui fonctionnent comme l'instrument de communication quotidien et privilégié d'une communauté. Il y a donc un risque non négligeable que des données recueillies dans de telles conditions incluent, de façon parfaitement aléatoire, des énoncés qui donnent l'impression d'illustrer des mécanismes typologiquement intéressants, alors que ces mécanismes n'ont jamais existé auparavant dans cette langue, et n'auraient peut-être aucune chance de se stabiliser si la langue reprenait un fonctionnement normal.

Comme je l'ai déjà dit ci-dessus, on ne peut pas faire de l'élicitation avec les derniers locuteurs d'une langue moribonde comme on le fait avec des locuteurs « normaux ». Et si on le fait quand même (ce qui est difficilement évitable, car tous les corpus ont leurs limites), la « description » qui en résultera sera d'une fiabilité douteuse. En effet, il n'est pas difficile de comprendre que les souvenirs d'un locuteur âgé qui ne pratique plus réellement sa langue sont particulièrement sujets à caution lorsqu'on les sollicite par un questionnement direct. Dans la perspective typologique, ceci est d'autant plus grave qu'aucune vérification ne sera possible dans l'avenir, et sur ce point, le fait de fixer toutes les données sous forme d'enregistrements ne peut pas apporter un correctif, puisque c'est la fiabilité même des consultants qui est en cause.

\subsubsection{Un problème institutionnel}

Dans les circonstances actuelles, les problèmes discutés dans ce qui précède se trouvent aggravés par une question d'ordre institutionnel. En effet, j'ai beaucoup insisté sur le fait qu'enquêter sur une langue à un stade avance de dépérissement est une entreprise beaucoup plus difficile et beaucoup plus aléatoire qu'enquêter sur une langue qui conserve une certaine vitalité. Outre les difficultés d'accès aux consultants (qui sont loin d'être négligeables), il est beaucoup plus difficile d'évaluer la fiabilité des données recueillies, et il est beaucoup plus facile de faire des erreurs dans l'interprétation des données. Autrement dit, dans un monde idéal, ce genre d'exercice devrait être réservé à des linguistes expérimentés ayant déjà fait leurs preuves en décrivant des langues, si possible proches génétiquement et/ou 
géographiquement de la langue à décrire, qui fonctionnent encore comme fonctionnent normalement les langues. Or c'est exactement le contraire qui s'observe. La raison est tout simplement que depuis une vingtaine d'années, il est beaucoup plus facile d'obtenir un financement pour décrire des langues en danger que pour décrire des langues ayant une bonne vitalité. On peut même dire que la chance d'obtenir un financement pour une recherche en linguistique descriptive est inversement proportionnelle au degré de vitalité de la langue qu'on a pour projet de décrire.

On peut comprendre les motivations des organismes qui sponsorisent ces recherches. On peut comprendre aussi que le problème se pose différemment dans d'autres perspectives. Par exemple, le recueil de données lexicales et/ou morphologiques destinées à être exploitées pour la comparaison génétique et la reconstruction ne pose pas des problèmes comparables. On peut aussi évoquer la perspective de la revitalisation : dans cette perspective, tout ce qui importe est de fournir aux néo-locuteurs des données leur permettant d'assurer une certaine continuité avec la langue parlée par les générations précédentes. Mais compte tenu de tout ce qui a été discuté ci-dessus, on peut admettre que du point de vue de l'utilisation future par les typologues, notamment par les spécialistes de typologie syntaxique, la description de langues engagées dans un processus de dépérissement pose un problème qu'à mon avis on a tort de laisser sous silence.

\section{CONCLUSION}

Dans cet article je me suis efforcé d'argumenter que dans la perspective d'une exploitation typologique des travaux sur les langues en danger, il convient de distinguer entre des langues en danger qui gardent une certaine vitalité, et d'autres à un stade déjà avancé de dépérissement. Les premières ne posent aucun problème particulier par rapport aux langues qu'il n'y a pas lieu de classer comme langues en danger, que ce soit du point de vue de la méthodologie de la description ou du point de vue de l'exploitation typologique des descriptions. Il n'y a aucune réserve à avoir en ce qui concerne l'importance de leur témoignage en ce qui concerne la diversité des structures linguistiques. Pour les autres par contre, quel que soit l'intérêt qu'il puisse y avoir à les décrire dans d'autres perspectives, il est permis d'avoir des doutes sur ce que leur description peut apporter à la typologie linguistique.

Autrement dit, dans la perspective du développement de la typologie linguistique (et spécifiquement dans cette perspective), il est crucial de développer prioritairement la description de langues qui sont encore l'instrument de communication quotidien et privilégié d'une communauté, mais qui du fait d'évolutions dans la 
société risquent de se trouver rapidement dans une situation bien plus précaire. En effet, dans l'immédiat, il n'y a aucun problème particulier à décrire ces langues et à intégrer leur description à des bases de données typologiques. Mais si on tarde trop à les décrire, elles risquent rapidement de se trouver dans une situation qui complique beaucoup leur description, et qui rend problématique l'utilisation de leur description dans une perspective typologique. Or il se trouve que cette situation est probablement celle d'une large majorité des langues actuellement attestées.

\section{RÉFÉRENCE}

Epps, Patience, 2010. « Linguistic typology and language documentation », Jae Jung Song (ed.), The Oxford Handbook of Linguistic Typology, Oxford, Oxford University Press, 634-649. 